\title{
Long non-coding RNA ELFN1-AS1 in the pathogenesis of pancreatic cancer
}

\author{
Gang Ma, Guichen Li, Anjiang Gou, Zhihuan Xiao, Yuanhong Xu, Shaowei Song, Kejian Guo, Zhe Liu \\ Department of Pancreatic-Biliary Surgery, First Hospital of China Medical University, Shenyang, China \\ Contributions: (I) Conception and design: G Ma, Z Liu; (II) Administrative support: None; (III) Provision of study materials or patients: None; (IV) \\ Collection and assembly of data: G Li; (V) Data analysis and interpretation: G Ma, Z Liu; (VI) Manuscript writing: All authors; (VII) Final approval \\ of manuscript: All authors. \\ Correspondence to: Dr. Zhe Liu. 155 North Nanjing St., Shenyang 110001, China. Email: liuzhe4321@126.com.
}

\begin{abstract}
Background: Long non-coding ribonucleic acid (lncRNA) ELFN1 antisense RNA 1 (ELFN1-AS1) is involved in the pathogenesis of many different cancers. But the current research on the relationship between lncRNA ELFN1-AS1 and pancreatic cancer is still blank.

Methods: We investigated the role of lncRNA ELFN1-AS1 in the pathogenesis of pancreatic cancer using bioinformatics, in vitro and in vivo experiments in pancreatic cancer cell lines, and surgically removed clinical samples.

Results: Through bio-information analysis and in vitro and in vivo experiments, we found that LncRNA ELFN1-AS1 was highly enriched in pancreatic cancer data sets and highly expressed in pancreatic cancer cell lines and tissues. The knocking down of lncRNA ELFN1-AS1 significantly increased cancer cell death and growth arrest. Xenografts in nude mice showed that the growth of SW1990 cells in the mice group with a stable knock down of lncRNA ELFN1-AS1 was significantly slower than that in the control group.

Conclusions: The experimental results show that the expression of LncRNA ELFN1-AS1 is related to the growth and invasion ability of pancreatic cancer cells. By further studying the function of LncRNA ELFN1AS1 in pancreatic cancer, LncRNA ELFN1-AS1 was found to be involved in the epithelial-mesenchymal transition process in pancreatic cancer.
\end{abstract}

Keywords: Bioinformatics; long non-coding RNA; pancreatic cancer; epithelial-mesenchymal transition

Submitted Mar 29, 2021. Accepted for publication May 21, 2021.

doi: $10.21037 / \mathrm{atm}-21-2376$

View this article at: http://dx.doi.org/10.21037/atm-21-2376

\section{Introduction}

Pancreatic cancer accounts for $3 \%$ of all cancers and $7 \%$ of all cancer-related deaths (1). A survey carried out between 2009 and 2015 found that the 5 -year survival rate of pancreatic cancer patients was only $9 \%$ (2). As it is often detected at a late stage, research has shown that only $20 \%$ of patients with pancreatic cancer have surgically removable lesions at diagnosis (3). Thus, a better understanding of the mechanisms of pathogenesis of pancreatic cancer is key to improved prognosis.

Long non-coding ribonucleic acids (lncRNAs) are longer than 200 nucleotides and are not translated into protein products (4). Emerging evidence has shown that lncRNAs are critical regulators (5) and prognostic biomarkers (6) of diverse cancers. Specifically, lncRNA ELFN1 antisense RNA 1 (ELFN1-AS1) has been found to be involved in the pathogenesis of esophageal cancer (7), ovarian cancer (8), colorectal cancer (9), retinoblastoma (10), and hepatocellular carcinoma (11).

In this study, we investigated the role of lncRNA ELFN1-AS1 in the pathogenesis and progression of pancreatic cancer using a bioinformatic analysis, and in vitro and in vivo experiments in different pancreatic cancer cell lines and surgically removed pancreatic cancer tissues. We found that lncRNA ELFN1-AS1 was highly expressed in publicly deposited online cancer databases, 
pancreatic cancer cell lines, and clinical tissues, and that the knocking down of lncRNA ELFN1-AS1 led to increased cancer cell death and arrested cell cycle. LncRNA ELFN1-AS1 was found to be involved in pancreatic cancer pathogenesis by promoting the epithelial-mesenchymal transition (EMT) process.

We present the following article in accordance with the ARRIVE reporting checklist (available at http://dx.doi. org/10.21037/atm-21-2376).

\section{Methods}

\section{Bioinformatics}

Transcriptome sequencing data were collected from The Cancer Genome Atlas (TCGA)-Pancreatic Adenocarcinoma (PAAD) data set using R package (TCGA biolinks). A differential microRNA (mRNA) abundance analysis was performed using DESeq2 (http://www.r-project.org/). Genes with reads $<5$ were not included in the quantitative analyses. Volcano plots were obtained based on the normalized gene expression using $\mathrm{R}$ package. Normalized gene expressions were analyzed by Gene Set Variation Analyses (GSVAs). R package survival was used for the survival analyses. A Cox proportional hazard (PH) model was calculated using function survival and survminer from $\mathrm{R}$ packages. The best-scanned cut-off points were defined as those with the most significant splits (according to log-rank tests). $\mathrm{R}$ package survival receiver operating characteristics (ROCs) were used for the ROC curves, and areas under the curve s(AUCs) were plotted for the survival analyses.

\section{Patients}

All procedures performed in this study involving human participants were in accordance with the Declaration of Helsinki (as revised in 2013). Ethical approval was obtained from the Ethical Committee of the First Affiliated Hospital of China Medical University (Shenyang, China) (No. [2015]100). Written informed consent was obtained from all enrolled patients. Each diagnosis of pancreatic cancer was made according to the National Comprehensive Cancer Network (NCCN, 2018) guidelines. No patient received preoperative chemotherapy. Pancreatic cancer tissues and adjacent normal tissue from the same patients were obtained during pancreaticoduodenectomies at the First Affiliated Hospital of China Medical University from June 1, 2018 to May 31, 2019. Patients were excluded if they had bacterial or viral infections, chronic diseases, including hypertension, cardiovascular diseases, diabetes, or other types of cancer. Tissue samples were cryopreserved in liquid nitrogen immediately after surgical resection until required.

\section{Cell culture}

All experiments were performed using mycoplasma-free cells. Human PAAD cell lines BxPC-3 (RRID:CVCL_0186), SW1990 (RRID:CVCL_1723), and PANC-1 (RRID:CVCL_0480) were purchased from Shanghai Zhong Qiao Xin Zhou Biothechnology Co., Ltd (Shanghai, China). Cells were cultured in Dulbecco's modified Eagle's medium (DMEM, Gibco, Thermo Fisher Scientific, Inc.) supplemented with $10 \%$ fetal bovine serum (FBS, Gibco), $10 \mathrm{mM}$ HEPES (Gibco), $2 \mathrm{mM}$ L-glutamine (Gibco), $1 \mathrm{mM}$ pyruvate sodium (Gibco), $100 \mathrm{U} / \mathrm{ml}$ penicillin, and $100 \mu \mathrm{g} / \mathrm{ml}$ streptomycin (Gibco) at $37^{\circ} \mathrm{C}$ with $5 \%$ carbon dioxide $\left(\mathrm{CO}_{2}\right)$.

\section{Construction of a stable lncRNA-knock-down cell line}

A specific SW1990 cell line (SW1990-LNC-KD) with the stable knock down of lncRNA ELFN1-AS1 and a scramble control SW1990 cell line were constructed with lnc-small hairpin RNA (shRNA) or a scramble control (see Table S1) cloned into the pLV3 (H1/GFP \& Puro) vector (see Figure S1) and transfected into 293T cells (Shanghai GenePharma China). Supernatants were collected after $72 \mathrm{~h}$ incubation and used for the transfection ( $24 \mathrm{~h}$ incubation before transfection) of the SW1990s (12). SW1990 cells containing the lnc-shRNA were screened by media containing $5 \mu \mathrm{g} / \mathrm{mL}$ puromycin (Sigma, St. Louis, MO, USA), and used for the Xenograft tumor growth experiments (13).

\section{Transfection of siRNAs}

Small interfering RNAs (siRNAs) and scrambled negative control cell lines for lncRNA were provided by Shanghai GenePharma (Shanghai, China). siRNAs (30 nM; see Table S1) were transfected with $\mathrm{X}$-tremeGENE siRNA transfection reagent (Roche Applied Science, Shanghai, China) in accordance with the manufacturer's instructions.

\section{Total RNA extraction and qRT-PCR}

Fresh cells or frozen tissues were homogenized in TRIzol reagent (Thermo Fisher Scientific, Inc.) for total RNA extraction in accordance with the manufacturer's 
instructions. RNA samples were quantified by NanoDrop 2000 Spectrophotometers (Thermo Fisher Scientific, Inc.) and reverse transcribed using an RT(Reverse transcription) reagent Kit (Nachuan Bio-Tech Co., Binzhou, China) in accordance with the manufacturer's instructions. A quantitative real-time polymerase chain reaction (qRTPCR) analysis was performed in an Exicycle 96 Real-Time Quantitative Thermal Block (Bioneer) using SYBR Green Master Mix (Nachuan Bio-Tech Co.) in accordance with the manufacturer's protocol (the sequence of primers is listed in Table S1). The average of the triplicate qRT-PCR results of the target IncRNA level from each sample was normalized by $\beta$-actin of the same sample, and the relative expression was calculated using the $2^{-\Delta \Delta \mathrm{Ct}}$ method.

\section{Cell count kit-8 assay for cell proliferation}

Cell proliferation was determined by a cell counting kit (CCK)-8 assay (DOJINDO, Japan). Cells were seeded into 96 -well plates $\left(1 \times 10^{4} /\right.$ well). Ten $\mu \mathrm{L}$ CCK-8 reagent in $100 \mu \mathrm{L}$ medium was aliquoted to each well. Light absorbance at $450 \mathrm{~nm}$ was measured after 2 hours of incubation at $37^{\circ} \mathrm{C}$ with an Epoch microplate spectrophotometer (BioTek China, Beijing). Cell viability was measured in triplicate experiments and expressed as a percentage of that of the control cells.

\section{Flow cytometry}

Forty-eight hours after siRNA transfection, cell death/ apoptosis was analyzed by flow cytometry (LSR, BD Biosciences) using an Annexin V-FITC/PI Apoptosis Detection Kit (Beyotime Institute of Biotechnology, Shanghai, China) in accordance with the manufacturer's instructions. Briefly, cells were collected, washed 3 times with cold phosphate-buffered saline (PBS), and stained in $500 \mu \mathrm{L}$ staining buffer (Annexin V-FITC/PI in PBS) at room temperature for $30 \mathrm{~min}$ in the dark.

For the cell-cycle analysis, the cells were collected, washed 3 times with cold PBS, fixed in precooled anhydrous ethanol at $4{ }^{\circ} \mathrm{C}$ for $30 \mathrm{~min}$, and stained with $50 \mu \mathrm{g} / \mathrm{mL}$ Propidium Iodide (PI; Solarbio Biotech, China) in $500 \mu \mathrm{L}$ PBS in the dark before being analyzed by flow cytometry. All flow cytometry experiments were triplicated.

\section{Immunofluorescence staining}

Cells were grown on sterile glass slides, fixed with $4 \%$ paraformaldehyde for $30 \mathrm{~min}$, blocked with $1 \%$ BSA for $30 \mathrm{~min}$, incubated with primary antibodies against E-cadherin CDH1 (AF0131, 1:500; Affinity Biosciences Ltd.), N-cadherin (AF4039, 1:200; Affinity Biosciences Ltd.), and Vimentin (AF7013, 1:250; Affinity Biosciences Ltd.) at $4{ }^{\circ} \mathrm{C}$ overnight, and then incubated with Alexa Fluor $^{\circledR} 594$ conjugated Affinipure Goat Anti-Rabbit IgG $(\mathrm{H}+\mathrm{L})$ secondary antibodies (Jackson ImmunoResearch Laboratories, PA) for 1 hour. Nuclei were counterstained with DAPI (4',6-diamidino-2-phenylindole). Images were captured with Olympus IX81 inverted fluorescence microscope (Olympus, Beijing, China).

\section{Western blotting}

Anti-beta actin (AF7018, 1:3,000), anti-E-cadherin (AF0131, 1:10,000), anti-Vimentin (AF7013, 1:1,000), anti$\mathrm{N}$-cadherin (AF4039, 1:1,000), and the secondary antibody (S0001, 1:5,000) were obtained from Affinity Biosciences Ltd. 48 hours after siRNA transfection, the cells were lysed using RIPA lysis buffer (Merck Group, Germany) for $30 \mathrm{~min}$ on ice. Sample protein concentrations were quantified using a BCA (Bicinchoninic Acid) assay kit (Solarbio, China). Equal amounts of proteins were separated by SDS-PAGE (sodium dodecyl sulfate polyacrylamide gel electrophoresis), transferred to polyvinylidene difluoride membranes, and probed with the antibodies of interest. The intensity of bands was quantified by ImageJ, using $\beta$-actin as the internal loading control.

\section{Xenograft tumor growth in nude mice}

All animal experiments were approved by the Ethics Committee of the First Affiliated Hospital of China Medical University (No: [2015]100), and performed according to the National Institutes of Health Guide for the Care and Use of Laboratory Animals. Male nude mice were kept in a temperature-controlled specific-pathogen-free animal laboratory on a $12 \mathrm{~h}$ light/dark cycle with free access to food and water. SW1990-LNC-KD cells $\left(1.5 \times 10^{6}\right.$ cells in $0.1 \mathrm{~mL}$ sterile PBS, with stable knock down of lncRNA ELFN1AS1), or SW1990-LNC-NC cells (with scrambled control shRNA) were subcutaneously injected into the left flanks of the mice at 8 weeks of age. Tumor volume was measured in the morning by length $\mathrm{x}$ width $\mathrm{x}$ depth in $\mathrm{mm}$. The mice were euthanized 2 weeks after the cancer cell injection, and the subcutaneous tumors were surgically removed and compared ( $\mathrm{n}=7$ in each group). 


\section{Immunobistochemistry}

Immunohistochemical staining was performed on $10 \mu \mathrm{m}$ sections of paraffin-embedded tissues. Slides were incubated with proliferating cell nuclear antigen (PCNA) antibody (AF0239, Affinity Biosciences Ltd.; at 1:100 dilution) at $4{ }^{\circ} \mathrm{C}$ overnight, followed by a secondary antibody (S0001, 1:200 dilution, Affinity Biosciences Ltd.) at $37^{\circ} \mathrm{C}$ for 1 hour, and counterstained with hematoxylin. Positive cells were quantified by IHC Profiler (14).

\section{Transwell assay}

SW1990 $\left(1 \times 10^{5}\right)$ or BxPC-3 $\left(1 \times 10^{5}\right)$ cells were resuspended in $100 \mu \mathrm{L}$ serum-free medium 24 hours after transfection and seeded into the upper chamber with $12 \mu \mathrm{m}$ pore polycarbonate membranes pre-coated with Matrigel Basement Membrane Matrix (BD Biosciences, Bedford, MA, USA). The lower chamber was filled with $600 \mu \mathrm{L}$ of 1640 medium supplemented with $20 \%$ FBS. After 24 hours of incubation (at $37{ }^{\circ} \mathrm{C}$ with $5 \%\left(\mathrm{CO}_{2}\right.$ ), the invaded cells were fixed and stained with $0.5 \%$ crystal violet (15).

\section{Wound-healing assay}

Cells were seeded in 24 -well plates at $1 \times 10^{5}$ cells/well 24 hours after transfection, in medium containing 5\% FBS and $5 \% \mathrm{CO}_{2}$ for $24 \mathrm{~h}$. A $1-\mathrm{mm}$ wide scratch was made in the confluent cultures with a pipette tip and washed twice with PBS to remove debris. The area of the scratch was measured using images taken by a phase-contrast microscope (16).

\section{Statistics}

The statistical analysis was performed using SPSS software (version 24, Armonk, NY, USA: IBM Corp.). Quantitative data are presented as mean \pm standard deviation (SD). Differences in the means of the 2 samples were analyzed using Student's $t$-tests. All 2-sided statistical tests were considered significant if $\mathrm{P}<0.05$.

\section{Results}

\section{LncRNA ELFN1-AS1 expression in PAAD}

Bioinformatics data on PAAD transcriptome-level 3 (including 176 pancreatic cancer tissues and 4 normal pancreatic tissues) were obtained from the TCGA database (17) to identify the lncRNAs most relevant to pancreatic cancer. The DESeq2 software package (18) analysis showed that the expression of lncRNA ELFN1-AS1 was significantly higher than that of normal pancreatic tissues (see Figure 1A). Patients in the TCGA-PAAD cohort were further divided into a high-lncRNA ELFN1-AS1 group and a low-lncRNA ELFN1-AS1 group based on the median value. Using the GEPIA online tool (19) and a R-language survival analysis, patients in the high-lncRNA ELFN1-AS1 expression group were found to have a shorter survival period than those in the low-IncRNA ELFN1-AS1 group $(\mathrm{P}<0.05$; see Figure $1 B, C)$. Pancreatic cancer tissues $(\mathrm{n}=26)$ and selfpaired adjacent normal tissue from the same patients $(n=26)$ were obtained from 14 male and 12 female admitted patients, aged between 43 and 63 (mean age $53.8 \pm 6.0$ years) and analyzed by qRT-PCR. LncRNA ELFN1-AS1 levels were significantly higher in cancer tissues than normal tissues $(\mathrm{P}<0.01$; see Figure $1 D)$. The results clearly showed that IncRNA ELFN1-AS1 was highly expressed in pancreatic cancer tissues, and the overexpression of IncRNA ELFN1-AS1 could indicate a poor prognosis.

\section{LncRNA ELFN1-AS1 knock down inbibited PAAD cell growth, invasion, and migration}

A qRT-PCR analysis was performed to determine the expression of lncRNA ELFN1-AS1 in pancreatic cancer cell lines SW1990, PANC-1, and BxPC-3 cells. LncRNA ELFN1-AS1 was significantly higher in SW1990 and BxPC-3 cells than PANC-1 cells (see Figure $2 A$ ). We then used specific siRNAs to knock down the expression of lncRNA ELFN1-AS1 to study the function of lncRNA ELFN1-AS1 and found that siRNA1 was suitable for the subsequent knock-down experiments $(\mathrm{P}<0.05$; Figure $2 B)$. The CCK-8 assay showed that cell proliferation in the ELFN1-AS1 siRNA knock-down group was significantly lower than that in the control group in both SW1990 (n=3) and BxPC-3 (n=5) cell lines (see Figure 2C). Transwell (see Figure 2D) and wound-healing (see Figure $2 E$ ) experiments showed that the knocking down of lncRNA ELFN1-AS1 significantly inhibited cell migration and invasion, and significantly promoted cell death (see Figure $2 F$ ) where the cells in the knock-down group accumulated in the G1/S phase (Figure 2G). Thus, the knocking down of ELFN1AS1 inhibited growth, invasion, migration, and promoted apoptosis in PAAD cells. 
A

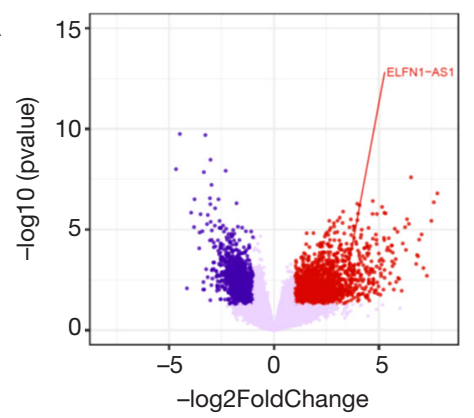

C

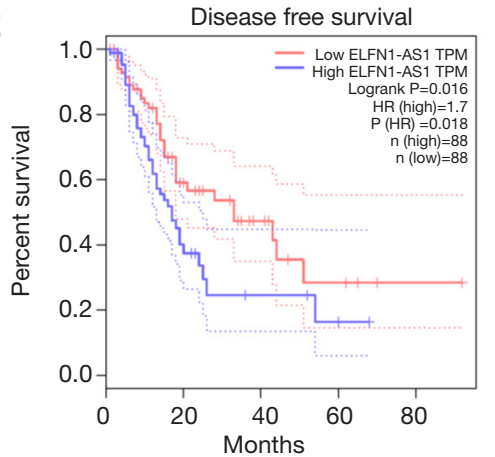

B



$\mathrm{D}$

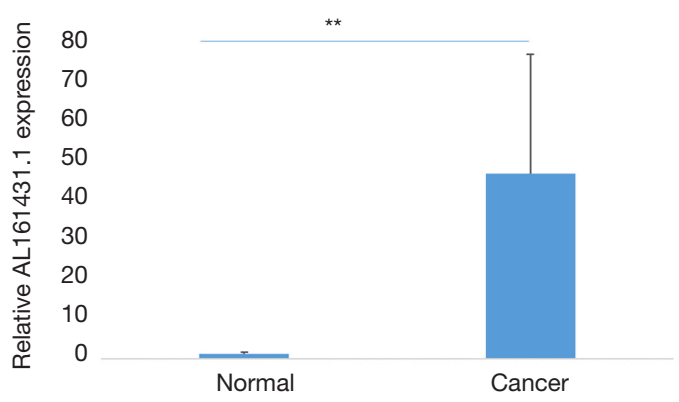

Figure 1 LncRNA ELFN1-AS1 was upregulated in pancreatic adenomas and indicated a poor prognosis. (A) A volcano plot of different gene expressions in TCGA-PAAD; Kaplan-Meier curves of the overall survival (B) and disease-free survival (C) of patients in the TCGAPAAD cohort; (D) relative AL161431.1 expression in pancreatic cancer and normal tissues from clinical samples ( $\mathrm{n}=24$, qRT-PCR). ${ }^{* *} \mathrm{P}<0.01$.

\section{LncRNA ELFN1-AS1 xenografts in nude mice}

To further evaluate the role of ELFN1-AS1 in vivo, we constructed a lncRNA ELFN1-AS1 stable knock-down cell line (SW1990-LNC-KD cells) with a specific shRNA. The cells in the 5th passage were found to have a minimum level of lncRNA ELFN1-AS1 (as confirmed by a qRTPCR analysis, data not shown), and were injected into nude mice. Two weeks after injection, the xenograft tumor size in the knock-down group was significantly smaller than that of the control group (see Figure $3 A$ ). The knockdown group also showed a much slower tumor growth at different checkpoints (see Figure 3B), a lower expression of lncRNA ELFN1-AS1 in the tumor tissue $(\mathrm{P}<0.01$; see Figure $3 C$ ), and reduced cell atypia and fewer PCNApositive cells in hematoxylin and eosin staining and PCNA (cell proliferation index) immunohistochemical staining (see Figure 3D). There was significantly fewer PCNA-positive cells in the SW1990-LNC-KD xenograft tumor tissue $(\mathrm{P}<0.01$; see Figure $3 E)$. The above results suggest that IncRNA ELFN1-AS1 knock down inhibited the growth of xenograft tumors.

\section{LncRNA ELFN1-AS1 function in pancreatic cancer}

To determine the function of lncRNA ELFN1-AS1, the median of lncRNA ELFN1-AS1 was used as the cut-off value threshold, and the expression of differential genes was analyzed by DEseq2 (see Figure $4 A$ ). The Gene Ontology (GO) (20) function analysis (see Figure 4B) and Kyoto Encyclopedia of Genes and Genomes (KEGG) analysis (see Figure 4C) of lncRNA ELFN1-AS1 showed that ELFN1-AS1 was involved in various cellular functions and processes, such as the cell-cell junction and extracellular microenvironment. We identified an interaction network conducted by IncRNA ELFN1-AS1 (see Figure $5 A$ ). Additionally, the GSVA (Gene Set Variation Analysis) analysis showed the differential genes of the high-lncRNA ELFN1-AS1 expression groups were closely associated with the EMT gene set (see Figure 5B,C), which was involved in cell migration and invasion. These bioinformatic analyses suggested that lncRNA ELFN1-AS1 could potentially regulate the migration and invasion of pancreatic cancer cells through the EMT process.

To further verify the above-mentioned bioinformatic 


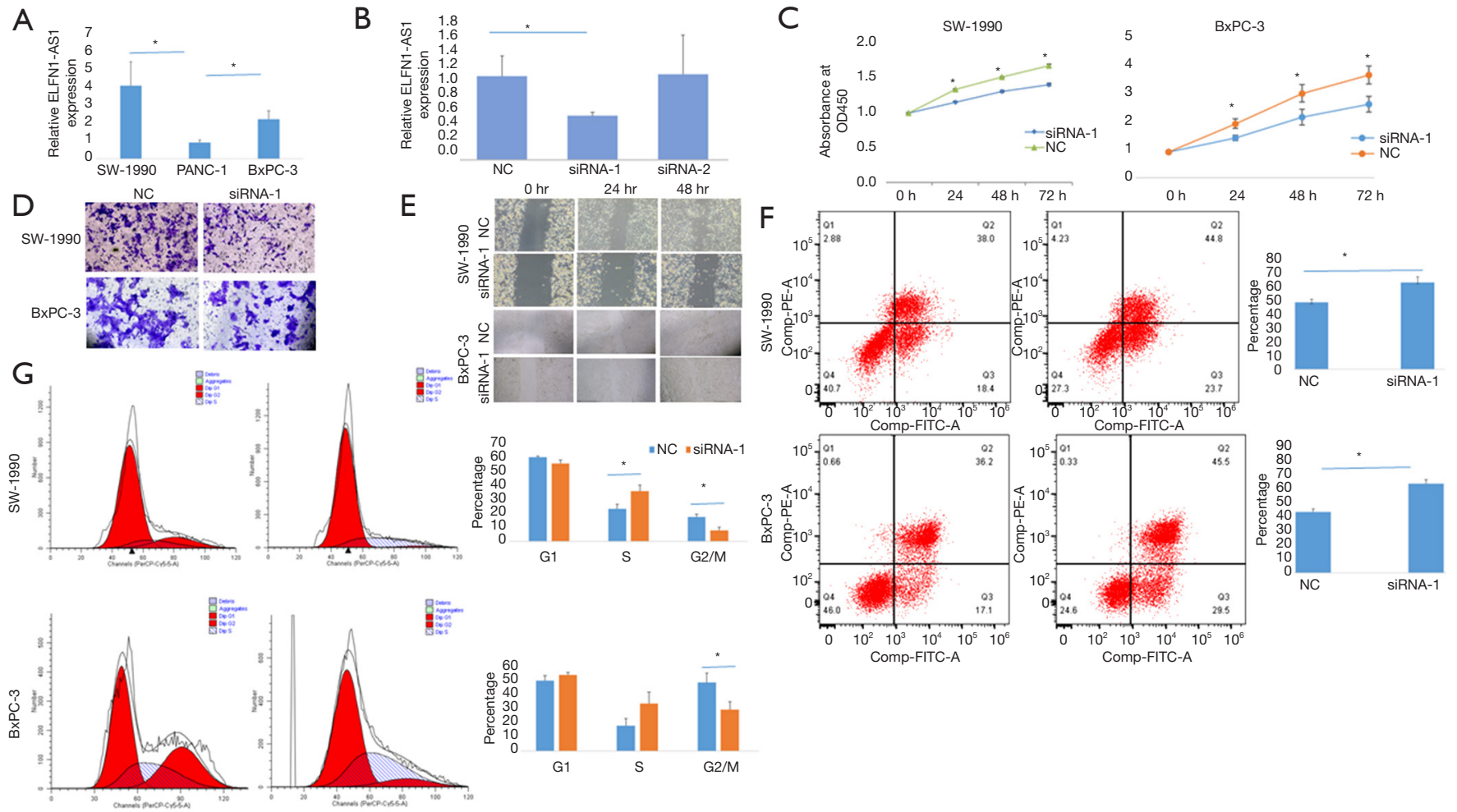

Figure 2 Knock down of lncRNA ELFN1-AS1 inhibited growth, invasion, and migration, and promoted apoptosis in PAAD cell lines. (A) Relative lncRNA ELFN1-AS1 expression in SW1990, PANC-1, and BxPC-3 cells analyzed by qRT-PCR; (B) relative lncRNA ELFN1AS1 expression in SW1990 cells after siRNA transfection analyzed by qRT-PCR; (C) a CCK-8 assay showing fold change of absorbance at OD450, representing a growth curve of SW1990 and BxPC-3 cells at 24, 48, and 72 hours after siRNA1 transfection; (D) transwell assay in scrambled control siRNA or siRNA1 transfected SW-1990 or BxPC-3 cells, stained with $0.5 \%$ crystal violet (100x); (E) wound-healing assay in scramble siRNA, or siRNA1 transfected SW1990 or BxPC-3 cells at 0, 24, and 48 hours post scratching (100×); (F) cell death (Q2+Q3) in scrambled control siRNA or siRNA1 transfected SW1990 and BxPC-3 cells analyzed by flow cytometry; (G) cell-cycle analysis of SW1990 and BxPC-3 cells after the transfection of scrambled control siRNA or siRNA1 by flow cytometry. NC, scrambled control siRNA. *P<0.05.

findings on the role of lncRNA ELFN1-AS1 in cell migration and invasion, we knocked down the expression of lncRNA ELFN1-AS1 with siRNA1 in both SW1990 and BxPC-3 cells. We found a significant increase in the expression of E-cadherin, and a decrease in the expression of $\mathrm{N}$-cadherin and vimentin in both mRNA and protein level after knocking down of lncRNA ELFN1-AS1, as confirmed by the qRT-PCR (see Figure $6 A$ ), Western blot (see Figure $6 B$ ) and immunofluorescence (se Figure $6 C$ ) analyses. These results suggested that lncRNA ELFN1-AS1 promoted cell migration and invasion by promoting the EMT process.

\section{Discussion}

LncRNAs are involved in the pathogenesis of pancreatic cancer. Most lncRNAs promote the proliferation of pancreatic cancer through different signaling pathways $(21,22)$. With the help of bioinformatics, we can not only identify candidate lncRNAs, but also lncRNAs-associated signaling pathways in advanced mechanistic studies. By analyzing the TCGA-PAAD dataset, we found that lncRNA ELFN1-AS1 was significantly increased in clinical pancreatic cancer tissues, and in pancreatic cancer cell lines SW1990 and BxPC-3. We validated bioinformatics findings using siRNA or shRNA to knock-down lncRNA ELFN1AS1 in those cells and found significantly more cell death and cell-cycle arrest. Interestingly, the pathway of lncRNA ELFN1-AS1 involved in the pathogenesis of pancreatic cancer was not the same as the previously reported microRNA pathway in other types of cancer (7-11); rather, the pathway involved in the pathogenesis of pancreatic cancer was the EMT pathway. 

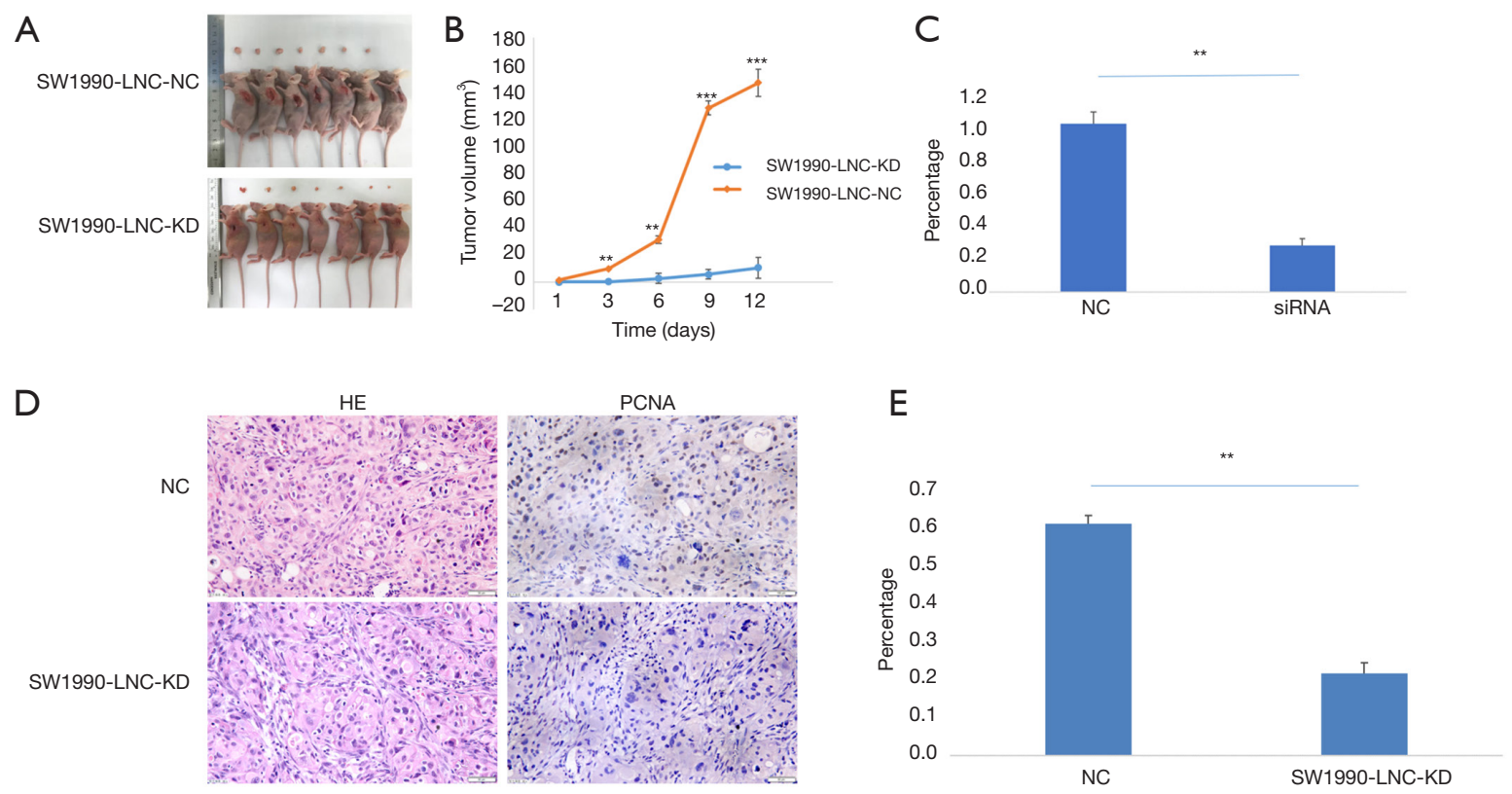

Figure 3 Knock down of lncRNA ELFN1-AS1 inhibited the growth of tumor xenografts. (A) Size of SW1990 tumor xenografts with SW1990-LNC-KD or SW1990-LNC-NC cells at 14 days after injection; (B) time course of in vivo xenografts growth measured in mm3; (C) relative AL161431.1 expression in engrafted tumors with SW1990-LNC-NC cell or SW1990-LNC-KD cell; (D) HE staining and PCNA immunohistochemistry of tumor xenograft sections, 100x; (E) relative ratio of PCNA-positive cells in the engrafted tumors with SW1990LNC-NC cell or SW1990-LNC-KD cell. ${ }^{* *} \mathrm{P}<0.01,{ }^{* * *} \mathrm{P}<0.001$.
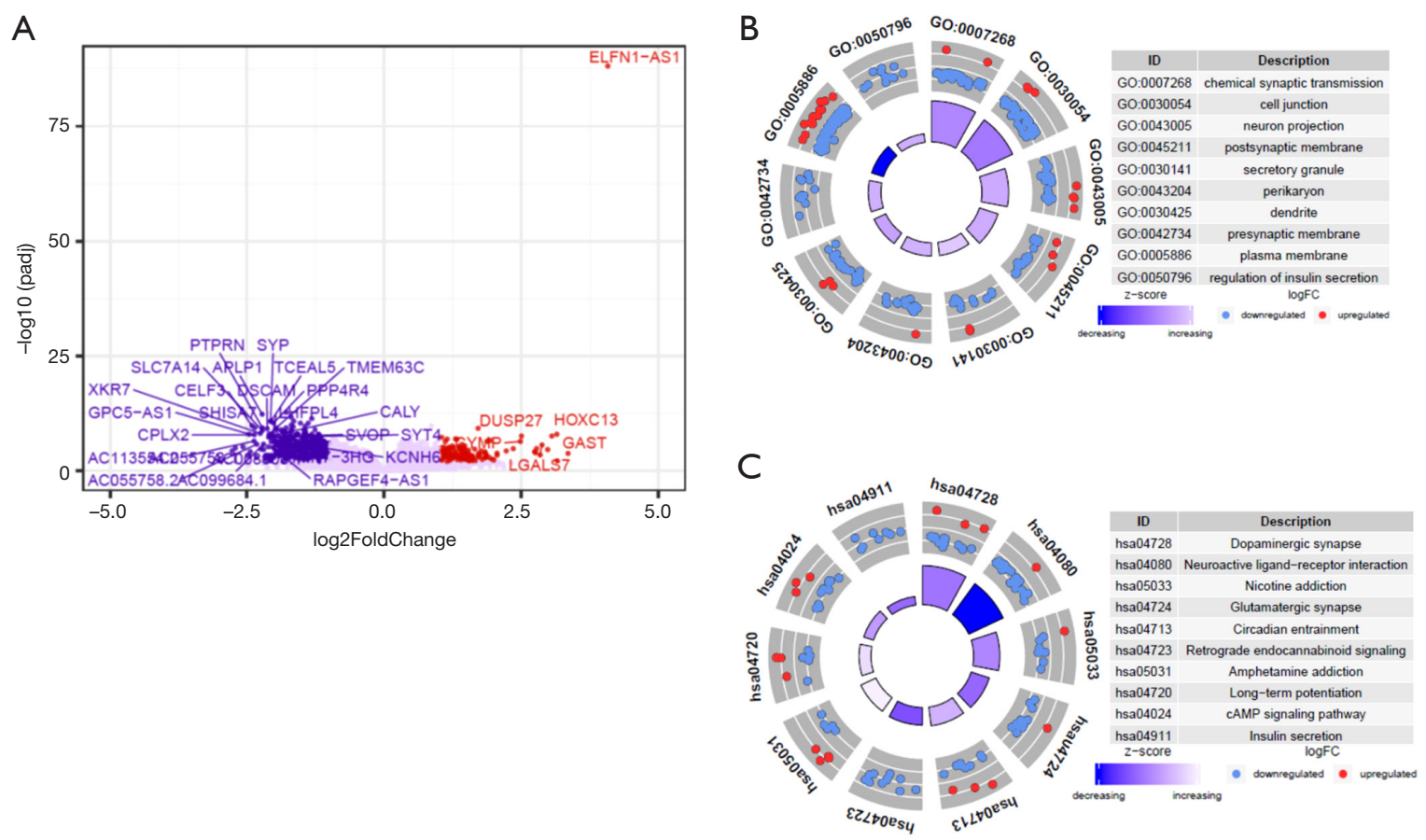

Figure 4 Prediction of the function of lncRNA ELFN1-AS1 in pancreatic adenomas. (A) A volcano plot of different gene expressions between with higher ELFN1-AS1 expression and lower ELFN1-AS1 expression patients in the TCGA-PAAD cohort; (B) GO function analysis of ELFN1-AS1; (C) KEGG analysis of ELFN1-AS1. 

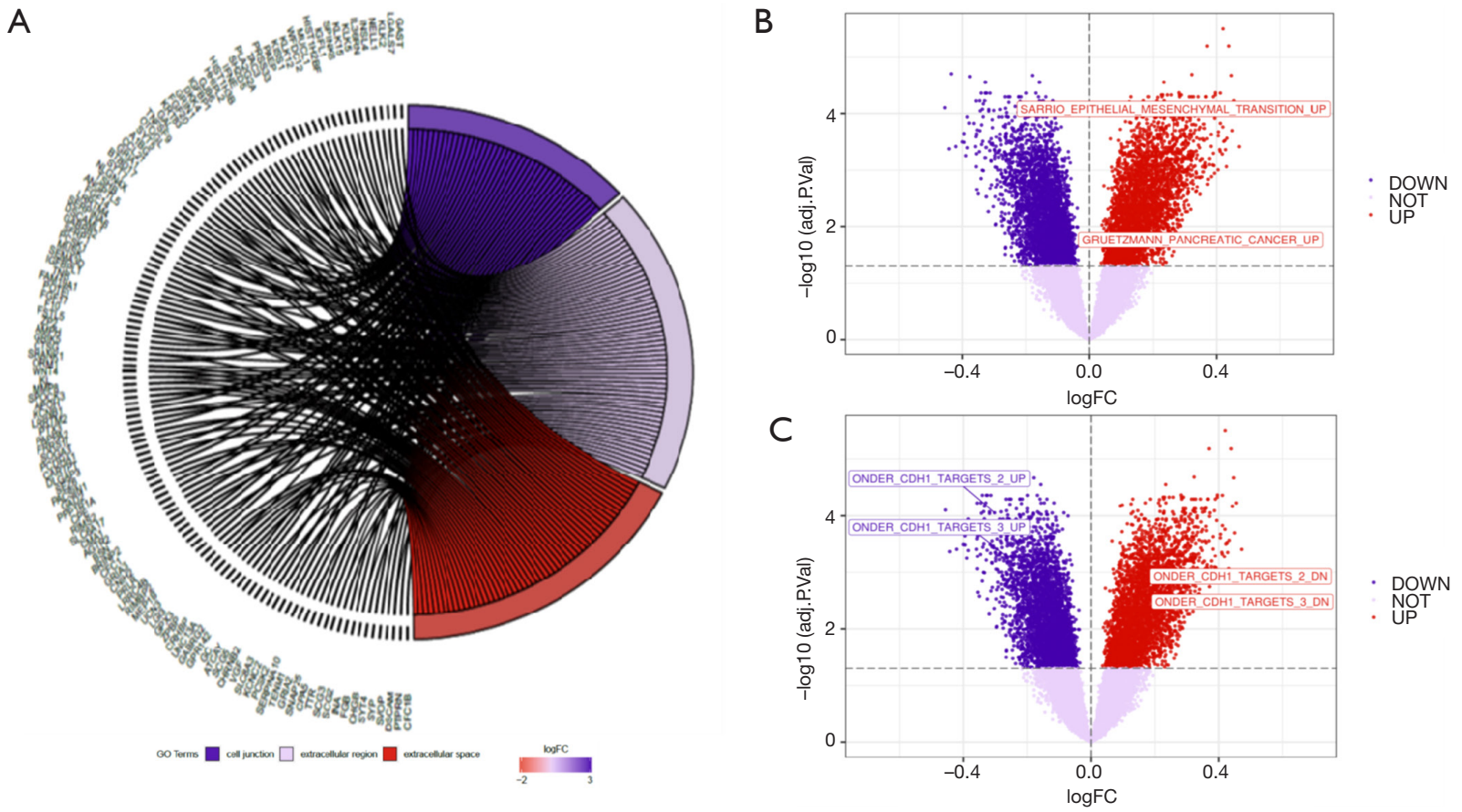

Figure 5 Mechanism of lncRNA ELFN1-AS1 in regulating cell function. (A) GO function analysis of lncRNA ELFN1-AS1; (B,C) GSVA analysis of different gene expressions in patients with high- and low-LncRNA ELFN1-AS1 in the TCGA-PAAD cohort. CDH1 is also known as E-cadherin.

A
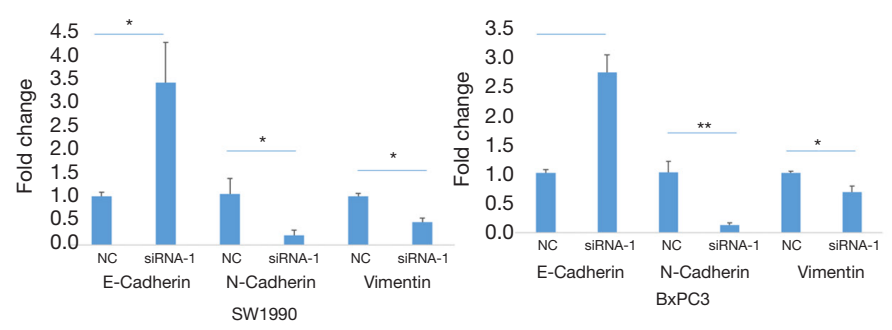

C

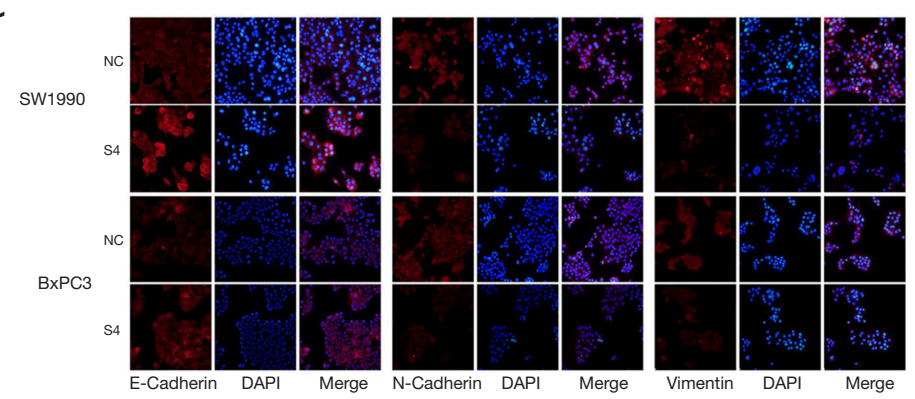

B
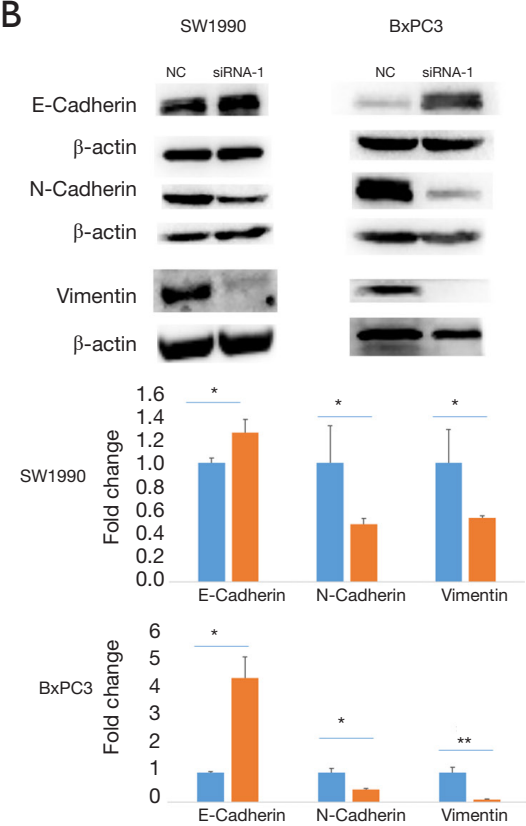

Figure 6 Knock down of lncRNA ELFN1-AS1 inhibited EMT. (A) qRT-PCR showing the knock down of lncRNA ELFN1-AS1, and the corresponding changes in E-cadherin, N-cadherin, and vimentin in SW1990 and BxPC-3 cells; (B) western blots showing the changes in protein level in E-cadherin, N-cadherin, and vimentin in SW1990 and BxPC-3 cells after transfection of scrambled siRNA or siRNA1; (C) immunofluorescence analysis showing the change of expression of E-cadherin, N-cadherin, and vimentin in SW1990 and BxPC-3 cells after transfection of scrambled siRNA or siRNA1 (200x). ${ }^{*} \mathrm{P}<0.05,{ }^{* *} \mathrm{P}<0.01$. 
EMT is an essential step in tumorigenesis, which enhances the metastasis and stemness of cancer cells. The canonical milestone of the EMT process is the increase of vimentin and $\mathrm{N}$-cadherin and the decrease of $\mathrm{E}$-cadherin. Regulating factors of EMT include the MAPK, TGF- $\beta$, JAK/STAT, Hedgehog, Wnt, and Hippo-YAP/TAZ signaling pathways (23). E-cadherin is critical in the initiation and maintenance of EMT, the cleavage of which leads to the destabilization of cell-cell junctions and the release of $\beta$-catenin for cell proliferation (24). An increase in vimentin and a decrease in E-cadherin has been reported in lung cancer cells (25). Vimentin is a key intermediate filament protein in normal mesenchymal tissue (26) that sustains cellular integrity (27). Additionally, vimentin is an independent adverse prognostic biomarker in patients with pancreatic ductal adenocarcinoma (28). The coexistence of increased $\mathrm{E}$-cadherin and decreased $\mathrm{N}$-cadherin and vimentin in the lncRNA ELFN1-AS1 knock-down pancreatic cancer cells in our study indicated that lncRNA ELFN1-AS1 is critical in the EMT process of pancreatic cancer.

In summary, we found that lncRNA ELFN1-AS1 was involved in the development and progression of pancreatic cancer by promoting the EMT process. LncRNA ELFN1AS1 might be a reliable prognostic predictor of and treatment target in pancreatic cancer.

\section{Acknowledgments}

Funding: This work is supported by Liaoning Provincial Department of Education Science Research Project (No. L2014299) and National Natural Science Foundation of China (No. 81572360).

\section{Footnote}

Reporting Checklist: The authors have completed the ARRIVE reporting checklist. Available at http://dx.doi. org/10.21037/atm-21-2376

Data Sharing Statement: Available at http://dx.doi. org/10.21037/atm-21-2376

Conflicts of Interest: All authors have completed the ICMJE uniform disclosure form (available at http://dx.doi. org/10.21037/atm-21-2376). The authors report funding from Liaoning Provincial Department of Education Science Research Project (No. L2014299) and National Natural
Science Foundation of China (No. 81572360). The authors have no other conflicts of interest to declare.

Ethical Statement: The authors are accountable for all aspects of the work in ensuring that questions related to the accuracy or integrity of any part of the work are appropriately investigated and resolved. All procedures performed in this study involving human participants were in accordance with the Declaration of Helsinki (as revised in 2013). Ethical approval for this study was obtained from the Ethical Committee of the First Affiliated Hospital of China Medical University (No. [2015]100). Written informed consent was obtained from all patients at admission. All animal experiments were approved by the Ethics Committee of the First Affiliated Hospital of China Medical University (No: [2015]100), and performed according to the National Institutes of Health Guide for the Care and Use of Laboratory Animals.

Open Access Statement: This is an Open Access article distributed in accordance with the Creative Commons Attribution-NonCommercial-NoDerivs 4.0 International License (CC BY-NC-ND 4.0), which permits the noncommercial replication and distribution of the article with the strict proviso that no changes or edits are made and the original work is properly cited (including links to both the formal publication through the relevant DOI and the license). See: https://creativecommons.org/licenses/by-nc-nd/4.0/.

\section{References}

1. The American Cancer Society medical and editorial content team. (2019, February 11). About Pancreatic Cancer. Retrieved July 20, 2020, Available online: https://www.cancer.org/content/dam/CRC/PDF/ Public/8778.00.pdf

2. The American Cancer Society medical and editorial content team. (2020, January 8). Survival Rates for Pancreatic Cancer. Retrieved July 20, 2020 Available online: https://www.cancer.org/cancer/pancreatic-cancer/ detection-diagnosis-staging/survival-rates.html

3. Saad AM, Turk T, Al-Husseini MJ, et al. Trends in pancreatic adenocarcinoma incidence and mortality in the United States in the last four decades; a SEER-based study. BMC Cancer 2018;18:688.

4. Engreitz JM, Haines JE, Perez EM, et al. Local regulation of gene expression by lncRNA promoters, transcription and splicing. Nature 2016;539:452-5. 
5. Wang S, Liang $\mathrm{K}, \mathrm{Hu} \mathrm{Q}$, et al. JAK2-binding long noncoding RNA promotes breast cancer brain metastasis. J Clin Invest 2017;127:4498-515.

6. Huang X, Zhi X, Gao Y, et al. LncRNAs in pancreatic cancer. Oncotarget 2016;7:57379-90.

7. Zhang C, Lian H, Xie L, et al. LncRNA ELFN1AS1 promotes esophageal cancer progression by upregulating GFPT1 via sponging miR-183-3p. Biol Chem 2020;401:1053-61.

8. Jie Y, Ye L, Chen H, et al. ELFN1-AS1 accelerates cell proliferation, invasion and migration via regulating miR497-3p/CLDN4 axis in ovarian cancer. Bioengineered 2020;11:872-82.

9. Lei R, Feng L, Hong D. ELFN1-AS1 accelerates the proliferation and migration of colorectal cancer via regulation of miR-4644/TRIM44 axis. Cancer Biomark 2020;27:433-43.

10. Feng W, Zhu R, Ma J, et al. LncRNA ELFN1-AS1 Promotes Retinoblastoma Growth and Invasion via Regulating miR-4270/SBK1 Axis. Cancer Manag Res 2021;13:1067-73.

11. Jia Y, Chen Y, Liu J. Prognosis-Predictive Signature and Nomogram Based on Autophagy-Related Long Noncoding RNAs for Hepatocellular Carcinoma. Front Genet 2020;11:608668.

12. Wang Y, Gao R, Li J, et al. Circular RNA hsa_ circ_0003141 promotes tumorigenesis of hepatocellular carcinoma via a miR-1827/UBAP2 axis. Aging (Albany NY) 2020;12:9793-806.

13. Wei L, Ye H, Li G, et al. Cancer-associated fibroblasts promote progression and gemcitabine resistance via the SDF-1/SATB-1 pathway in pancreatic cancer. Cell Death Dis 2018;9:1065.

14. Varghese F, Bukhari AB, Malhotra R, et al. IHC Profiler: an open source plugin for the quantitative evaluation and automated scoring of immunohistochemistry images of human tissue samples. PLoS One 2014;9:e96801.

15. Pijuan J, Barcelo C, Moreno DF, et al. In vitro Cell Migration, Invasion, and Adhesion Assays: From Cell Imaging to Data Analysis. Front Cell Dev Biol 2019;7:107.

16. Jonkman JE, Cathcart JA, Xu F, et al. An introduction to the wound healing assay using live-cell microscopy. Cell Adh Migr 2014;8:440-51.

17. Genomic Data Commons Data Portal. Retrieved July 20, 2020. Available online: https://portal.gdc.cancer.gov/ projects/TCGA-PAAD
18. Bioconductor. Differential gene expression analysis based on the negative binomial distribution. Retrieved July 20, 2020. Available online: https://bioconductor.org/packages/ release/bioc/html/DESeq2.html

19. Tang Z, Li C, Kang B, et al. GEPIA: a web server for cancer and normal gene expression profiling and interactive analyses. Nucleic Acids Res 2017;45:W98-102.

20. The Gene Ontology C. The Gene Ontology Resource: 20 years and still GOing strong. Nucleic Acids Res 2019;47:D330-8.

21. Chen L, Zhang J, Chen Q, et al. Long noncoding RNA SOX2OT promotes the proliferation of pancreatic cancer by binding to FUS. Int J Cancer 2020;147:175-88.

22. Lu Y, Tang L, Zhang Z, et al. Long Noncoding RNA TUG1/miR-29c Axis Affects Cell Proliferation, Invasion, and Migration in Human Pancreatic Cancer. Dis Markers 2018;2018:6857042.

23. Loh CY, Chai JY, Tang TF, et al. The E-Cadherin and $\mathrm{N}-$ Cadherin Switch in Epithelial-to-Mesenchymal Transition: Signaling, Therapeutic Implications, and Challenges. Cells 2019;8:1118.

24. Niehrs C. The complex world of WNT receptor signalling. Nat Rev Mol Cell Biol 2012;13:767-79.

25. Chen HH, Zhou XL, Shi YL, et al. Roles of p38 MAPK and JNK in TGF-beta1-induced human alveolar epithelial to mesenchymal transition. Arch Med Res 2013;44:93-8.

26. Leader M, Collins M, Patel J, et al. Vimentin: an evaluation of its role as a tumour marker. Histopathology 1987;11:63-72.

27. Fuchs E, Weber K. Intermediate filaments: structure, dynamics, function, and disease. Annu Rev Biochem 1994;63:345-82.

28. Myoteri D, Dellaportas D, Lykoudis PM, et al. Prognostic Evaluation of Vimentin Expression in Correlation with Ki67 and CD44 in Surgically Resected Pancreatic Ductal Adenocarcinoma. Gastroenterol Res Pract 2017;2017:9207616.

(English Language Editor: L. Huleatt)

Cite this article as: Ma G, Li G, Gou A, Xiao Z, Xu Y, Song S, Guo K, Liu Z. Long non-coding RNA ELFN1-AS1 in the pathogenesis of pancreatic cancer. Ann Transl Med 2021;9(10):877. doi: 10.21037/atm-21-2376 


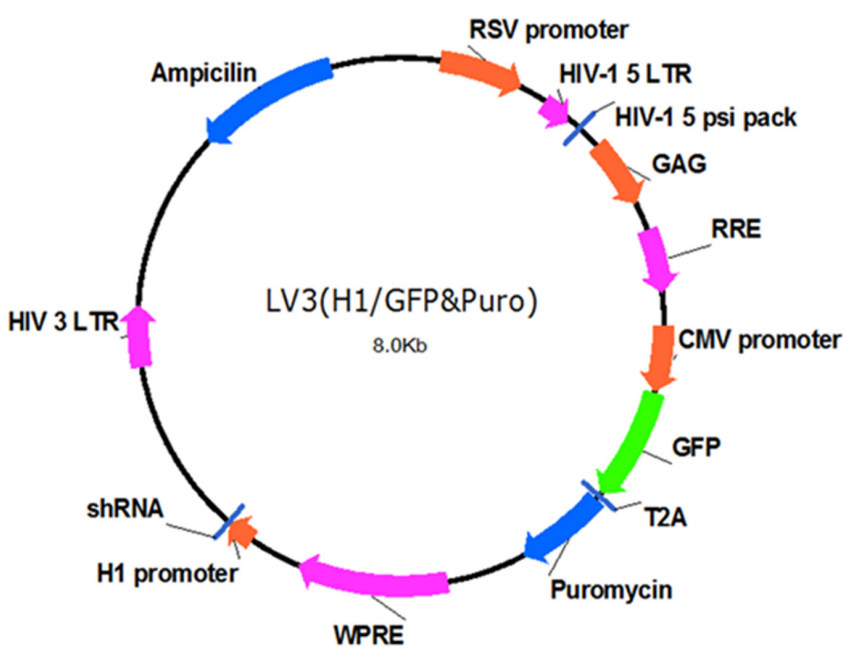

Figure S1 Map of shRNA vector.

Table S1 Primers for qRT-PCR and shRNA/siRNAs

\begin{tabular}{lll}
\hline ELFN1-AS1 & Forward (qRT-PCR) & 5'-ACCATCCGCCACATTCCTAC-3' \\
\hline ELFN1-AS1 & Reverse (qRT-PCR) & 5'-GCAGGTGGATTAGATGCTGC-3' \\
siRNA-1 & ELFN1-AS1 & Sense: 5'-GCAUCUAAUCCACCUGCAGTT-3'; antisense: \\
& 5'-CUGCAGGUGGAUUAGAUGCTT-3' \\
siRNA-2 & ELFN1-AS1 & sense: 5'-GCCUCAGCCACAAUCGUAATT-3'; antisense: \\
& 5'-CUGCAGGUGGAUUAGAUGCTT-3' \\
siRNA-NC & Sense: 5'-UUCUCCGAACGUGUCACGUTT-3'; antisense: \\
& & 5'-ACGUGACACGUUCGGAGAATT-3' \\
Lnc-shRNA & 5'-GCAUCUAAUCCACCUGCAGTT-3' \\
Scramble shRNA & 5'-TTCTCCGAACGTGTCACGT-3' \\
\hline
\end{tabular}

